\title{
Well posedness of a nonlinear mixed problem for a parabolic equation with integral condition
}

\author{
Abdelkader Djerad $^{1 *}$ (D, Ameur Memou² and Ali Hameida ${ }^{3}$
}

\section{"Correspondence:}

abdelkader.djerad@univ-msila.dz

${ }^{1}$ Laboratoire de Mathematiques

Pures et Appliquées, Department of

Mathematics, University of Msila,

Msila, Algeria 28000

Full list of author information is

available at the end of the article

\begin{abstract}
The aim of this work is to prove the well posedness of some posed linear and nonlinear mixed problems with integral conditions. First, an a priori estimate is established for the associated linear problem and the density of the operator range generated by the considered problem is proved by using the functional analysis method. Subsequently, by applying an iterative process based on the obtained results for the linear problem, the existence, uniqueness of the weak solution of the nonlinear problems is established.
\end{abstract}

MSC: 35B45; 35K61

Keywords: Energy inequality; Integral boundary conditions; Strong solution; Weak solution; Second order parabolic equation

\section{Introduction and statement of the problem}

Some problems related to physical and technical issues can be effectively described in terms of nonlocal problems with integral conditions in partial differential equations. These nonlocal conditions arise mainly when the values on the boundary cannot be measured directly, while their average values are known. The problem of parabolic equation with integral condition is stated as follows: Let us consider the rectangular domain $Q=] 0,1[\times] 0, T[$, then the problem is to find a solution $\sigma(x, t)$ of the following nonclassical boundary value problem:

$$
\left.£ \sigma=\frac{\partial \sigma}{\partial t}-\frac{\partial}{\partial x}\left(a \frac{\partial \sigma}{\partial x}\right)=g\left(x, t, \sigma, \frac{\partial \sigma}{\partial x}\right), \quad \text { for }(x, t) \in\right] 0,1[\times] 0, T[
$$

with the initial condition

$$
l \sigma=\sigma(x, 0)=\varphi(x), \quad \text { for } x \in[0,1],
$$

and the Dirichlet boundary condition

$$
\sigma(0, t)=0, \quad \text { for } t \in[0, T]
$$

(c) The Author(s) 2021. This article is licensed under a Creative Commons Attribution 4.0 International License, which permits use, sharing, adaptation, distribution and reproduction in any medium or format, as long as you give appropriate credit to the original author(s) and the source, provide a link to the Creative Commons licence, and indicate if changes were made. The images or other third party material in this article are included in the article's Creative Commons licence, unless indicated otherwise in a credit line to the material. If material is not included in the article's Creative Commons licence and your intended use is not permitted by statutory regulation or exceeds the permitted use, you will need to obtain permission directly from the copyright holder. To view a copy of this licence, visit http://creativecommons.org/licenses/by/4.0/. 
and the nonlocal condition

$$
\int_{0}^{\alpha} \sigma(x, t) d x+\int_{\beta}^{1} \sigma(x, t) d x=0, \quad 0 \leq \alpha \leq \beta<1 \forall t \in[0, T] .
$$

In addition, we assume that the function $a(x, t)$ and its derivatives satisfy the conditions

$$
\begin{cases}0<a_{0} \leq a(x, t) \leq a_{1} & \forall(x, t) \in Q \\ c_{2} \leq \frac{\partial a}{\partial t}(x, t) \leq c_{1}, & \forall(x, t) \in Q \\ \left|\frac{\partial a}{\partial x}(x, t)\right| \leq b, & \forall(x, t) \in Q\end{cases}
$$

where the functions $g\left(x, t, \sigma, \frac{\partial \sigma}{\partial x}\right), \varphi(x)$ are given, and we assume that the following matching conditions are satisfied:

$$
\left\{\begin{array}{l}
\varphi(0)=0, \\
\int_{0}^{\alpha} \varphi(x) d x+\int_{\beta}^{1} \varphi(x) d x=0 .
\end{array}\right.
$$

We also assume that there exists a positive constant $d$ such that

$$
\left|g\left(x, t, \sigma_{1}, \frac{\partial \sigma_{1}}{\partial x}\right)-g\left(x, t, \sigma_{2}, \frac{\partial \sigma_{2}}{\partial x}\right)\right| \leq d\left(\left|\sigma_{1}-\sigma_{2}\right|+\left|\frac{\partial \sigma_{1}}{\partial x}-\frac{\partial \sigma_{2}}{\partial x}\right|\right),
$$

for all $(x, t) \in Q$.

This type of problem can be found in various physic problems such as heat conduction [1-4], plasma physics [5], thermoelasticity [6], electrochemistry [7], chemical diffusion [8] and underground water flow [9-11]. Several research papers such as found in [1-4, 7, 1218] have studied and solved the parabolic equation by combining the integral condition with Dirichlet condition or Newmann condition, or with purely integral conditions, using various methods. For hyperbolic equations, the unicity and existence of the solution have been studies in [13,19-22] and the mixed-type equations in [23-27]. The elliptic equations were considered in [28,29] and [30].

The linear problem associated to the problem stated in (1.1)-(1.4), for $\alpha=\beta=0$, has been studied in [18] and for $\beta=1$ in [16]. Meanwhile in [31] the solved problem is for the case $\alpha+\beta=1$. It is worth mentioning that in [32] the author studied the same case where $\frac{\partial}{\partial x}\left(a \frac{\partial \sigma}{\partial x}\right)$ was replaced by the Bessel operator.

In the present paper the motivation is to study and find a solution to the stated problem without imposing any conditions on the constants $\alpha$ and $\beta$ in the interval $[0,1]$. In addition, the nonlinear problem of the parabolic equation with integral condition defined on two parts of the boundary is solved.

First, an a priori estimate is established for the associated linear problem and the density of the operator range generated by the considered problem is proved by using the functional analysis method. Subsequently, by applying an iterative process based on the obtained results for the linear problem, the existence and uniqueness of the weak solution of the nonlinear problems is established.

The rest of the paper is organized as follows. In Sect. 2, the associated linear problem is stated. Section 3 deals with the proof of the uniqueness of the solution using an a priori 
estimate. Section 4 gives the solvability of the considered linear problem. Finally, in Sect. 5, on the basis of the obtained results in Sects. 3 and 4, and on the use of an iterative process, we prove the existence and uniqueness of the solution of the nonlinear problem.

\section{Statement of the associated linear problem}

In this section we introduce the linear problem and the different function spaces needed to investigate the mixed nonlocal problem given by the equation.

$$
£ u=\frac{\partial u}{\partial t}-\frac{\partial}{\partial x}\left(a \frac{\partial u}{\partial x}\right)=f(x, t)
$$

and the conditions given by (1.2)-(1.4).

The given problem (2.1), (1.2)-(1.4) can be considered as finding a solution of the operator equation $L u=(£ u, l u)=\mathcal{F}=(f, \varphi)$, where the operator $L$ has as a domain of definition $D(L)$ consisting of functions $u \in L^{2}(Q)$ such that $\frac{\partial u}{\partial t}, \frac{\partial u}{\partial x}, \frac{\partial^{2} u}{\partial x \partial t}(x, t) \in L^{2}(Q)$ and satisfying the conditions (1.3) and (1.4).

The operator $L$ is an operator acting on $E$ into $\mathbb{F}$, where $E$ is the Banach space of functions $u \in L^{2}(Q)$, with a finite norm

$$
\|u\|_{E}^{2}=\int_{Q}(1-x)^{2}\left[\left|\frac{\partial u}{\partial t}\right|^{2}+\left|\frac{\partial^{2} u}{\partial x^{2}}\right|^{2}\right] d x d t+\sup _{t} \int_{0}^{1}\left[(1-x)^{2}\left|\frac{\partial u}{\partial x}\right|^{2}+|u|^{2}\right] d x .
$$

$\mathbb{F}$ is the Hilbert space of functions $F=(f, \varphi), f \in L^{2}(Q), \varphi \in H^{1}(0,1)$ with the finite norm

$$
\|F\|_{\mathbb{F}}^{2}=\int_{Q}(1-x)^{2}|f(x, t)|^{2} d x d t+\int_{0}^{1}\left[(1-x)^{2}\left|\frac{d \varphi}{d x}\right|^{2}+|\varphi|^{2}\right] d x .
$$

Then we show that the operator $L$ has a closure $\bar{L}$ and later on, in Sect. 3, we establish an energy inequality of the following type (see Theorem 3.1):

$$
\|u\|_{E} \leq k\|L u\|_{\mathbb{F}} \quad \forall u \in D(L) .
$$

Definition 2.1 A solution of the operator equation $\bar{L} u=F=(f, \varphi)$ is called a strong solution of problem (2.1)-(1.4).

Since the points of the graph of the operator $\bar{L}$ are limits of sequences of points of the graph of $L$, we can extend the a priori estimate (2.4) to be applied to strong solutions by taking the limits, that is, we have the inequality

$$
\|u\|_{E} \leq c\|\bar{L} u\|_{\mathbb{F}}, \quad \forall u \in D(\bar{L}) .
$$

From this inequality, we deduce the uniqueness of a strong solution, if it exists, and that the range of the operator $\bar{L}$ coincides with the closure of the range of $L$.

Proposition 2.1 The operator $L: E \longrightarrow F$ admits a closure $\bar{L}$.

Proof Let $u_{n} \in D(L)$ be a sequence such that

$$
\lim _{n \rightarrow \infty} u_{n}=0 \quad \text { in } E
$$


and

$$
\lim _{n \rightarrow \infty} L u_{n}=F=(f, \varphi) \quad \text { in the space } F
$$

we then must show that $f=0, \varphi=0$.

Since (2.6) holds, we have

$$
\lim _{n \rightarrow \infty} u_{n}=0 \quad \text { in } D^{\prime}(Q),
$$

where $D^{\prime}(Q)$ is the space of distribution on $Q$. By virtue of the continuity of derivation of $D^{\prime}(Q)$ in $D^{\prime}(Q),(2.8)$ implies that

$$
\lim _{n \rightarrow \infty} £ u_{n}=0 \quad \text { in } D^{\prime}(Q)
$$

According to (2.7), we have

$$
\lim _{n \rightarrow \infty} £ u_{n}=f \quad \text { in } L_{\rho}^{2}(Q),
$$

where $L_{\rho}^{2}(Q)$ is a Banach space with norm $\|u\|_{L_{\rho}^{2}(Q)}^{2}=\int_{Q} \frac{(1-x)^{2}}{2}|u|^{2} d x d t$. Then

$$
\lim _{n \rightarrow \infty} £ u_{n}=f \quad \text { in } D^{\prime}(Q) .
$$

By virtue of the uniqueness of the limit in $D^{\prime}(Q)$, we conclude that $f=0$.

According to (2.7), we also conclude that

$$
\lim _{n \rightarrow \infty} l u_{n}=\varphi \quad \text { in } H_{\rho}^{1}(0,1)
$$

where $H_{\rho}^{1}(0,1)$ is a Banach space with norm $\|u\|_{H_{\rho}^{1}(0,1)}^{2}=\int_{0}^{1}\left(\frac{(1-x)^{2}}{2}\left|\frac{\partial u}{\partial x}\right|^{2}+|u|^{2}\right) d x$. By the fact that the canonical injection from $H_{\rho}^{1}(0,1)$ into $D^{\prime}(0,1)$ is continuous, we deduce that

$$
\lim _{n \rightarrow \infty} l u_{n}=\varphi \quad \text { in } D^{\prime}(0,1)
$$

Moreover, since (2.6) holds and

$$
\left\|l u_{n}\right\|_{H_{\rho}^{1}(0,1)} \leq\left\|u_{n}\right\|_{E}
$$

we have

$$
\lim _{n \rightarrow \infty} l u_{n}=0 \quad \text { in } H_{\rho}^{1}(0,1),
$$

Hence

$$
\lim _{n \rightarrow \infty} l u_{n}=0 \quad \text { in } D^{\prime}(0,1),
$$

By virtue of the uniqueness of the limit in $D^{\prime}(0,1)$, we conclude, from (2.10) and (2.11), that $\varphi=0$. This proves Proposition 2.1 
The following a priori estimate gives the uniqueness of the solution of the posed linear problem.

\section{An energy inequality and its application}

In this section, the uniqueness of the solution will be proved using an energy inequality method.

Theorem 3.1 There exists a positive constant $K$, such that for each function $u \in D(L)$ we have

$$
\|u\|_{E} \leq K\|L u\|_{\mathbb{F}}
$$

Proof Let

$$
M u=\lambda \frac{(1-x)^{2}}{2} \frac{\partial u}{\partial t}+\lambda(1-x) \int_{0}^{x} \frac{\partial u}{\partial t} d \zeta-x e^{\delta(x-1)} \int_{x}^{1} g(\zeta, t) d \zeta,
$$

where

$$
g(\zeta, t)=k \int_{0}^{\zeta} \frac{\partial u}{\partial t} d \mu-(k-\lambda) \int_{\alpha}^{\zeta} \frac{\partial u}{\partial t} d \mu+(k-\lambda) \int_{\beta}^{\zeta} \frac{\partial u}{\partial t} d \mu,
$$

and $\lambda, k$ and $\delta$ are a positives scalar parameters such that

$$
3<\frac{\lambda}{k}<4 e^{-\delta} \quad \text { with } \delta>\ln \left(\frac{4}{3}\right) .
$$

Taking the scalar product in $L^{2}\left(Q^{s}\right)$, where $Q^{s}=[0,1] \times[0, s]$ of Eq. $(2.1)$ and the operator $e^{-c t} \overline{M u}$, with $0 \leq s \leq T, c>0$, we have

$$
\begin{aligned}
\Phi(u, u) & =\operatorname{Re} \int_{Q^{s}} e^{-c t} f(x, t) \overline{M u} d x d t \\
& =\operatorname{Re} \int_{Q^{s}} e^{-c t} \frac{\partial u}{\partial t} \overline{M u} d x d t-\operatorname{Re} \int_{Q^{s}} e^{-c t} \frac{\partial}{\partial x}\left(a(x, t) \frac{\partial u}{\partial x}\right) \overline{M u} d x d t .
\end{aligned}
$$

Substituting $M u$ by its expression in the first term in the right-hand side of (3.3), we obtain

$$
\begin{aligned}
\operatorname{Re} \int_{Q^{s}} e^{-c t} \frac{\partial u}{\partial t} \overline{M u} d x d t= & \lambda \int_{Q^{s}} \frac{(1-x)^{2}}{2} e^{-c t}\left|\frac{\partial u}{\partial t}\right|^{2} d x d t \\
& -\operatorname{Re} \int_{Q^{s}} x e^{\delta(x-1)} e^{-c t} \frac{\partial u}{\partial t} \int_{x}^{1} \overline{g(\zeta, t)} d \zeta d x d t \\
& +\operatorname{Re} \int_{Q^{s}} \lambda(1-x) e^{-c t} \frac{\partial u}{\partial t} \overline{\int_{0}^{x} \frac{\partial u}{\partial t} d \zeta d x d t}
\end{aligned}
$$

Integrating by parts the second term in the right-hand side of the last equality of (3.4) with respect to $x$, using the fact that $\frac{\partial u}{\partial t}=\frac{1}{k} x e^{\delta(x-1)} \frac{\partial g}{\partial x}$, then

$$
\operatorname{Re} \int_{0}^{1} x e^{\delta(x-1)} \frac{\partial u}{\partial t} \int_{x}^{1} \overline{g(\zeta, t)} d \zeta d x=\frac{1}{k} \operatorname{Re} \int_{0}^{1} x e^{\delta(x-1)} \frac{\partial g}{\partial x} \int_{x}^{1} \overline{g(\zeta, t)} d \zeta d x
$$


Djerad et al. Boundary Value Problems

(2021) 2021:70

Page 6 of 24

integrating by parts with respect to $x$, we obtain

$$
\begin{aligned}
\operatorname{Re} & \int_{0}^{1} x e^{\delta(x-1)} \frac{\partial u}{\partial t} \int_{x}^{1} \overline{g(\zeta, t)} d \zeta d x \\
= & \frac{1}{k} \operatorname{Re} \int_{0}^{1} x e^{\delta(x-1)} \frac{\partial g}{\partial x} \int_{x}^{1} \overline{g(\zeta, t)} d \zeta d x \\
= & \left.\frac{1}{k} x e^{\delta(x-1)} g \int_{x}^{1} \overline{g(\zeta, t)} d \zeta d x\right|_{x=0} ^{x=1}+\frac{1}{k} \int_{Q^{s}} x e^{\delta(x-1)} e^{-c t}|g(x, t)|^{2} d x \\
& -\frac{1}{k} \operatorname{Re} \int_{0}^{1}(1+\delta x) e^{\delta(x-1)} g \int_{x}^{1} \overline{g(\zeta, t)} d \zeta d x,
\end{aligned}
$$

using this equality

$$
\begin{aligned}
\frac{d}{d x}|h(x)|^{2} & =\frac{d}{d x} h(x) \overline{h(x)}=\frac{d h(x)}{d x} \overline{h(x)}+h(x) \frac{d \overline{h(x)}}{d x} \\
& =\frac{d \overline{h(x)}}{d x} h(x)+h(x) \frac{d \overline{h(x)}}{d x}=2 \operatorname{Re}\left(h(x) \frac{d \overline{h(x)}}{d x}\right) .
\end{aligned}
$$

The last term in the previous equality becomes

$$
\begin{aligned}
-\frac{1}{k} & \operatorname{Re} \int_{0}^{1}(1+\delta x) e^{\delta(x-1)} g \int_{x}^{1} \overline{g(\zeta, t)} d \zeta d x \\
= & \frac{1}{2 k} \int_{0}^{1}(1+\delta x) e^{\delta(x-1)} \frac{d}{d x}\left|\int_{x}^{1} g(\zeta, t) d \zeta\right|^{2} d x \\
& +\frac{1}{2 k}\left(\left.(1+\delta x) e^{\delta(x-1)}\left|\int_{x}^{1} g(\zeta, t) d \zeta\right|^{2}\right|_{x=0} ^{x=1}\right. \\
& -\frac{1}{2 k} \int_{0}^{1}\left(2 \delta+\delta^{2} x\right) e^{\delta(x-1)}\left|\int_{x}^{1} g(\zeta, t) d \zeta\right|^{2} d x \\
= & -\frac{e^{-\delta}}{2 k} \int_{0}^{s} e^{-c t}\left|\int_{0}^{1} g(\zeta, t) d \zeta\right|^{2} d t-\frac{1}{2 k} \int_{0}^{1}\left(2 \delta+\delta^{2} x\right) e^{\delta(x-1)}\left|\int_{x}^{1} g(\zeta, t) d \zeta\right|^{2} d x .
\end{aligned}
$$

Then

$$
\begin{aligned}
\operatorname{Re} & \int_{Q^{s}} x e^{\delta(x-1)} e^{-c t} \frac{\partial u}{\partial t} \int_{x}^{1} \overline{g(\zeta, t)} d \zeta d x d t \\
= & \frac{1}{k} \operatorname{Re} \int_{Q^{s}} x e^{\delta(x-1)} e^{-c t} \frac{\partial g}{\partial x} \int_{x}^{1} \overline{g(\zeta, t)} d \zeta d x d t \\
= & \frac{1}{k} \int_{Q^{s}} x e^{\delta(x-1)} e^{-c t}|g(x, t)|^{2} d x d t-\frac{1}{2 k} \int_{Q^{s}}\left(2 \delta+\delta^{2} x\right) e^{\delta(x-1)} e^{-c t}\left|\int_{x}^{1} g(\zeta, t) d \zeta\right|^{2} d x d t \\
& -\frac{e^{-\delta}}{2 k} \int_{0}^{s} e^{-c t}\left|\int_{0}^{1} g(\zeta, t) d \zeta\right|^{2} d t .
\end{aligned}
$$


Similarly integrating by parts the last term of (3.4) with respect to $x$, we obtain

$$
\begin{aligned}
& \operatorname{Re} \int_{Q^{s}} \lambda(1-x) e^{-c t} \frac{\partial u}{\partial t} \overline{\int_{0}^{x} \frac{\partial u}{\partial t} d \zeta} d x d t \\
& =\frac{\lambda}{2 k^{2}} \int_{Q^{s}} e^{-c t}|g(x, t)|^{2} d x d t \\
& \quad+\frac{\lambda}{2 k^{2}} \int_{0}^{s} e^{-c t}|g(0, t)|^{2} d t-\frac{\lambda}{k^{2}} \operatorname{Re} \int_{Q^{s}} e^{-c t} g(0, t) \overline{g(x, t)} d x d t
\end{aligned}
$$

From (3.7) and (3.6), equality (3.4) becomes

$$
\begin{aligned}
\operatorname{Re} \int_{Q^{s}} e^{-c t} \frac{\partial u}{\partial t} \overline{M u} d x d t \\
=\lambda \int_{Q^{s}} \frac{(1-x)^{2}}{2} e^{-c t}\left|\frac{\partial u}{\partial t}\right|^{2} d x d t+\frac{\lambda}{2 k^{2}} \int_{Q^{s}} e^{-c t}|g(x, t)|^{2} d x d t \\
\quad+\frac{\lambda}{2 k^{2}} \int_{Q^{s}} e^{-c t}|g(0, t)|^{2} d x d t+\frac{e^{-\delta}}{2 k} \int_{0}^{s} e^{-c t}\left|\int_{0}^{1} g(\zeta, t) d \zeta\right|^{2} d t \\
\quad-\frac{1}{k} \int_{Q^{s}} x e^{\delta(x-1)} e^{-c t}|g(x, t)|^{2} d x d t \\
\quad+\frac{1}{2 k} \int_{Q^{s}}\left(2 \delta+\delta^{2} x\right) e^{\delta(x-1)} e^{-c t}\left|\int_{x}^{1} g(\zeta, t) d \zeta\right|^{2} d x d t \\
\quad-\frac{\lambda}{k^{2}} \operatorname{Re} \int_{Q^{s}} e^{-c t} g(0, t) \overline{g(x, t)} d x d t .
\end{aligned}
$$

Similarly, substituting $M u$ by its expression in the last term in the right-hand side of (3.3), integrating by parts with respect to $x$, using the Dirichlet condition (1.3) and the integral condition (1.4) we get

$$
\begin{aligned}
& -\operatorname{Re} \int_{Q^{s}} e^{-c t} \frac{\partial}{\partial x}\left(a(x, t) \frac{\partial u}{\partial x}\right) \overline{M u} d x d t \\
& =\lambda \operatorname{Re} \int_{Q^{s}} \frac{(1-x)^{2}}{2} e^{-c t} a(x, t) \frac{\partial u}{\partial x} \frac{\overline{\partial^{2} u}}{\partial x \partial t} d x d t \\
& \quad+\operatorname{Re} \int_{Q^{s}}\left(\lambda-k x e^{\delta(x-1)}\right) a(x, t) e^{-c t} u \frac{\overline{\partial u}}{\partial t} d x d t \\
& \quad+\lambda \operatorname{Re} \int_{Q^{s}} \frac{\partial a(x, t)}{\partial x} e^{-c t} u \int_{0}^{x} \frac{\partial u}{\partial t} d \zeta d x d t \\
& \quad-\operatorname{Re} \int_{Q^{s}}\left(x \frac{\partial a}{\partial x}+2 a(1+\delta x)\right) e^{\delta(x-1)} e^{-c t} u \overline{g(x, t)} d x d t \\
& \quad+\operatorname{Re} \int_{Q^{s}}\left((1+\delta x) \frac{\partial a}{\partial x}+\left(2 \delta+\delta^{2} x\right) a\right) e^{\delta(x-1)} e^{-c t} u \int_{x}^{1} \overline{g(\zeta, t)} d \zeta d x d t
\end{aligned}
$$


Integrating by parts the first two terms with respect to $t$ in (3.9), using the condition (1.2) we have

$$
\begin{aligned}
& \lambda \operatorname{Re} \int_{Q^{s}} \frac{(1-x)^{2}}{2} e^{-c t} a \frac{\partial u}{\partial x} \frac{\partial^{2} u}{\partial x \partial t} d x d t \\
&= \frac{\lambda}{2} \int_{Q^{S}}\left(c a-\frac{\partial a}{\partial t}\right) \frac{(1-x)^{2}}{2} e^{-c t}\left|\frac{\partial u}{\partial x}\right|^{2} d x d t \\
& \quad+\left.\frac{\lambda}{2} \int_{0}^{1} \frac{(1-x)^{2}}{2} e^{-c s} a(x, s)\left|\frac{\partial u}{\partial x}\right|^{2}\right|_{t=s} d x-\frac{\lambda}{2} \int_{0}^{1} \frac{(1-x)^{2}}{2} a(x, 0)\left|\frac{d \varphi}{d x}\right|^{2} d x, \\
& \operatorname{Re} \int_{Q^{s}}\left(\lambda-k x e^{\delta(x-1)}\right) e^{-c t} a(x, t) u \frac{\overline{\partial u}}{\partial t} d x d t \\
&=\int_{Q^{s}}\left(c a-\frac{\partial a}{\partial t}\right)\left(\lambda-k x e^{\delta(x-1)}\right) e^{-c t}|u|^{2} d x d t \\
& \quad+\left.\int_{0}^{1}\left(\lambda-k x e^{\delta(x-1)}\right) e^{-c s} a(x, s)|u|^{2}\right|_{t=s} d x-\int_{0}^{1}\left(\lambda-k x e^{\delta(x-1)}\right) a(x, 0)|\varphi|^{2} d x,
\end{aligned}
$$

then from the above equalities and equalities (3.8) and (3.9), (3.3) becomes

$$
\begin{aligned}
\frac{\lambda}{2} \int_{Q^{S}} & \frac{(1-x)^{2}}{2} e^{-c t}\left|\frac{\partial u}{\partial t}\right|^{2} d x d t+\frac{\lambda}{2} \int_{Q^{s}}\left(c a-\frac{\partial a}{\partial t}\right) \frac{(1-x)^{2}}{2} e^{-c t}\left|\frac{\partial u}{\partial x}\right|^{2} d x d t \\
& +\frac{1}{2 k} \int_{Q^{s}}\left(\frac{\lambda}{k}-2 x e^{\delta(x-1)}\right) e^{-c t}|g(x, t)|^{2} d x d t+\frac{\lambda}{2 k^{2}} \int_{Q^{s}} e^{-c t}|g(0, t)|^{2} d x d t \\
& +\frac{1}{2 k} e^{-\delta} \int_{0}^{s} e^{-c t}\left|\int_{0}^{1} g(x, t)\right|^{2} d x d t+\frac{1}{2} \int_{Q^{s}}\left(c a-\frac{\partial a}{\partial t}\right)\left(\lambda-k x e^{\delta(x-1)}\right) e^{-c t}|u|^{2} d x d t \\
& +\frac{1}{2 k} \int_{Q^{s}}\left(2 \delta+\delta^{2} x\right) e^{\delta(x-1)} e^{-c t}\left|\int_{x}^{1} g(\zeta, t) d \zeta\right|^{2} d x d t \\
& +\frac{\lambda}{2} \int_{0}^{1} \frac{(1-x)^{2}}{2} e^{-c s} a(x, s)\left|\frac{\partial u}{\partial x}\right|^{2}||_{t=s} d x \\
& +\left.\frac{1}{2} \int_{0}^{1}\left(\lambda-k x e^{\delta(x-1)}\right) e^{-c s} a(x, s)|u|^{2}\right|_{t=s} d x \\
& +\lambda \operatorname{Re} \int_{Q^{s}} \frac{\partial a(x, t)}{\partial x} e^{-c t} u \int_{0}^{x} \frac{\partial u}{\partial t} d \zeta d x d t \\
& -\operatorname{Re} \int_{Q^{s}}\left(x \frac{\partial a}{\partial x}+2 a(1+\delta x)\right) e^{\delta(x-1)} e^{-c t} u \overline{g(x, t)} d x d t \\
& +\frac{\lambda}{2 k^{2}} \operatorname{Re} \int_{0}^{s} e^{-c t} g(0, t) \int_{0}^{1} \bar{g}(x, t) d x d t \\
& +\operatorname{Re} \int_{Q^{s}}\left((1+\delta x) \frac{\partial a}{\partial x}+\left(2 \delta+\delta^{2} x\right) a\right) e^{\delta(x-1)} e^{-c t} u \int_{x}^{1} g(\zeta, t) d \zeta d x d t \\
= & \operatorname{Re} \int_{Q^{s}} e^{-c t} f \overline{M u} d x d t+\frac{\lambda}{2} \int_{0}^{1} \frac{(1-x)^{2}}{2} a(x, 0)\left|\frac{d \varphi}{d x}\right|^{2} d x \\
+ & \frac{1}{2} \int_{0}^{1}\left(\lambda-k x e^{\delta(x-1)}\right) a(x, 0)|\varphi|^{2} d x . \\
&
\end{aligned}
$$


Using the Young inequality in the last four terms in the left-hand side of (3.10), and using the facts that

$$
\int_{Q_{s}} e^{-c t}|u|^{2} d x d t \leq 8 \int_{Q^{s}} \frac{(1-x)^{2}}{2} e^{-c t}\left|\frac{\partial u}{\partial x}\right|^{2} d x d t
$$

and

$$
\int_{Q^{s}} e^{-c t}\left|\int_{0}^{x} \frac{\partial u}{\partial t} d \zeta\right|^{2} d x d t \leq 8 \int_{Q^{s}} \frac{(1-x)^{2}}{2} e^{-c t}\left|\frac{\partial u}{\partial t}\right|^{2} d x d t
$$

we get

$$
\begin{aligned}
& -\lambda \operatorname{Re} \int_{Q^{s}} e^{-c t} \frac{\partial a(x, t)}{\partial x} u \int_{0}^{x} \overline{\frac{\partial u}{\partial t} d \zeta} d x d t \\
& \leq 8 \lambda \epsilon_{1} b^{2} \int_{Q^{s}} \frac{(1-x)^{2}}{2} e^{-c t}\left|\frac{\partial u}{\partial x}\right|^{2} d x d t+\frac{2 \lambda}{\epsilon_{1}} \int_{Q^{s}} \frac{(1-x)^{2}}{2} e^{-c t}\left|\frac{\partial u}{\partial t}\right|^{2} d x d t, \\
& \operatorname{Re} \int_{Q^{s}}\left(x \frac{\partial a}{\partial x}+2 a(1+\delta x)\right) e^{-c t} u \overline{g(x, t)} d x d t \\
& \leq \frac{8\left(2 a_{1}(1+\delta)+b\right)^{2}}{2 \varepsilon_{2}} \int_{Q^{s}} \frac{(1-x)^{2}}{2} e^{-c t}\left|\frac{\partial u}{\partial x}\right|^{2} d x d t+\frac{\varepsilon_{2}}{2} \int_{Q^{s}} e^{-c t}|g(x, t)|^{2} d x d t, \\
& \operatorname{Re} \int_{Q^{s}}\left((1+\delta x) \frac{\partial a}{\partial x}+\left(2 \delta+\delta^{2} x\right) a\right) e^{\delta(x-1)} e^{-c t} u \int_{x}^{1} \overline{g(\zeta, t)} d \zeta d x d t \\
& \leq \frac{8\left(\left(2 \delta+\delta^{2}\right) a_{1}+(1+\delta) b\right)^{2}}{2 \varepsilon_{3}} \int_{Q^{s}} \frac{(1-x)^{2}}{2} e^{-c t}\left|\frac{\partial u}{\partial x}\right|^{2} d x d t \\
& +\frac{\varepsilon_{3}}{2} \int_{Q^{s}} e^{\delta(x-1)} e^{-c t}\left|\int_{x}^{1} \overline{g(\zeta, t)} d \zeta\right|^{2} d x d t, \\
& \frac{\lambda}{2 k^{2}} \operatorname{Re} \int_{0}^{s} g(0, t) \int_{0}^{1} \bar{g}(x, t) d x d t \leq \frac{\lambda \varepsilon_{4}}{4 k^{2}} \int_{0}^{s}|g(0, t)|^{2} d t+\frac{\lambda}{4 \varepsilon_{4} k^{2}} \int_{0}^{s}\left|\int_{0}^{1} g(x, t) d x\right|^{2} d t .
\end{aligned}
$$

We choose $\varepsilon_{1}=8, \varepsilon_{2}=2, \varepsilon_{3}=\frac{\delta}{k}$, and $\varepsilon_{4}=2$ and $c>0$ such that

$$
c>\frac{256 b^{2}}{a_{0}}+8 \frac{k}{\lambda a_{0}}\left(2 a_{1}(1+\delta)+b\right)^{2}+8 \frac{k}{\lambda \delta a_{0}}\left[\left(2 \delta+\delta^{2}\right) a_{1}+(1+\delta) b\right]^{2}+c_{2},
$$

therefore by combining the previous inequalities with (3.10), we get the following expression:

$$
\begin{aligned}
\frac{\lambda}{4} \int_{Q^{s}} & \frac{(1-x)^{2}}{2} e^{-c t}\left|\frac{\partial u}{\partial t}\right|^{2} d x d t+M \int_{Q^{s}} \frac{(1-x)^{2}}{2} e^{-c t}\left|\frac{\partial u}{\partial x}\right|^{2} d x d t \\
& +\frac{\lambda-k}{2}\left(c a_{0}-c_{2}\right) \int_{Q^{s}} e^{-c t}|u|^{2} d x d t+\left(\frac{\lambda}{2 k^{2}}-\frac{3}{2 k}\right) \int_{Q^{s}} e^{-c t}|g|^{2} d x d t \\
& +\left.\frac{\lambda a_{0}}{2} \int_{0}^{1} \frac{(1-x)^{2}}{2} e^{-c s}\left|\frac{\partial u}{\partial x}\right|^{2}\right|_{t=s} d x+\left.\lambda a_{0} \int_{0}^{1} e^{-c s}|u|^{2}\right|_{t=s} d x \\
\leq & \operatorname{Re} \int_{Q^{s}} e^{-c t} f \overline{M u} d x d t+\frac{\lambda a_{1}}{2} \int_{0}^{1} \frac{(1-x)^{2}}{2}\left|\frac{d \varphi}{d x}\right|^{2} d x+a_{1} \int_{0}^{1}|\varphi|^{2} d x,
\end{aligned}
$$


where

$$
M=\frac{\lambda}{2}\left(c a_{0}-c_{2}\right)-64 \lambda b^{2}-4 k\left(2 a_{1}(1+\delta)+b\right)^{2}-4 \frac{k}{\delta}\left[\left(2 \delta+\delta^{2}\right) a_{1}+(1+\delta) b\right]^{2} .
$$

Substituting $M u$ by its expression in the first term in the right-hand side of (3.12), we obtain

$$
\begin{aligned}
& \operatorname{Re} \int_{Q^{s}} e^{-c t} f \overline{M u} d x d t \\
& =\lambda \operatorname{Re} \int_{Q^{s}} \frac{(1-x)^{2}}{2} e^{-c t} f \frac{\overline{\partial u}}{\partial t} d x d t \\
& \quad+\lambda \operatorname{Re} \int_{Q^{s}}(1-x) e^{-c t} f \int_{0}^{x} \frac{\overline{\partial u}}{\partial t} d x d t+\operatorname{Re} \int_{Q^{s}} e^{-c t} x f \int_{x}^{1} \overline{g(x, \zeta)} d \zeta d x d t,
\end{aligned}
$$

each term in the right-hand side of (3.13), can be, respectively, controlled by

$$
\begin{aligned}
& \lambda \operatorname{Re} \int_{Q^{s}} e^{-c t} \frac{(1-x)^{2}}{2} f \frac{\overline{\partial u}}{\partial t} d x d t \\
& \quad \leq 2 \lambda \int_{Q^{s}} \frac{(1-x)^{2}}{2} e^{-c t}|f|^{2} d x d t+\frac{\lambda}{8} \int_{Q^{s}} \frac{(1-x)^{2}}{2} e^{-c t}\left|\frac{\partial u}{\partial t}\right|^{2} d x d t, \\
& \lambda \operatorname{Re} \int_{Q^{s}}(1-x) e^{-c t} f \int_{0}^{x} \frac{\overline{\partial u}}{\partial t} d x d t \\
& \quad \leq \frac{\lambda}{16} \int_{Q^{s}} e^{-c t} \frac{(1-x)^{2}}{2}\left|\frac{\partial u}{\partial t}\right|^{2} d x d t+64 \lambda \int_{Q^{s}} e^{-c t} \frac{(1-x)^{2}}{2}|f|^{2} d x d t,
\end{aligned}
$$

and

$$
\begin{aligned}
\operatorname{Re} & \int_{Q^{s}} x e^{\delta(x-1)} e^{-c t} f \int_{x}^{1} \overline{g(x, \zeta)} d \zeta d x d t \\
\leq & \frac{\lambda}{32} \int_{Q^{s}} \frac{(1-x)^{2}}{2} e^{-c t}\left|\frac{\partial u}{\partial t}\right|^{2} d x d t\left(\frac{\lambda^{2}-3 k}{4 k^{2}}\right) \int_{Q^{s}} \exp (-c t) \mid g\left(x,\left.t\right|^{2} d x d t\right. \\
& \quad+\left(\frac{2 k^{2}}{\lambda^{2}-3 k}+\frac{k^{2}}{128 \lambda}\right) \int_{Q^{s}} \exp (-c t) \frac{(1-x)^{2}}{2}|f|^{2} d x d t .
\end{aligned}
$$

The combination of the previous inequalities with (3.12) yields

$$
\begin{aligned}
& \int_{Q^{s}} \frac{(1-x)^{2}}{2}\left|\frac{\partial u}{\partial t}\right|^{2} d x d t+\int_{\Omega^{s}} \frac{(1-x)^{2}}{2}\left|\frac{\partial u}{\partial x}\right|^{2} d x d t \\
& \quad+\left.\int_{0}^{1} \frac{(1-x)^{2}}{2}\left|\frac{\partial u}{\partial x}\right|^{2}\right|_{t=s} d x+\left.\int_{0}^{1}|u|^{2}\right|_{t=s} d x \\
& \leq \sigma\left(\int_{Q} \frac{(1-x)^{2}}{2}|f|^{2} d x d t+\int_{0}^{1}\left(\frac{(1-x)^{2}}{2}\left|\frac{\partial \varphi}{\partial x}\right|^{2}+|\varphi|^{2}\right) d x\right)
\end{aligned}
$$

where

$$
\sigma=\frac{\max \left(\left(\frac{2 k^{2}}{\lambda^{2}-3 k}+\frac{k^{2}}{128 \lambda}+66 \lambda\right), \frac{\lambda a_{1}}{2}\right)}{\min \left(M, \frac{\lambda-k}{2} e^{-\delta}\left(c a_{0}-c_{2}\right), \frac{\lambda}{32}, \frac{\lambda-k}{2} e^{-\delta} a_{0}, \frac{\lambda a_{0}}{2}\right)} \exp (c T) .
$$


From Eqs. (1.1) and (3.15), we deduce that

$$
\begin{aligned}
\int_{Q^{s}} & \frac{(1-x)^{2}}{2}\left[\left|\frac{\partial u}{\partial t}\right|^{2}+\left|\frac{\partial^{2} u}{\partial x^{2}}\right|^{2}\right] d x d t+\int_{\Omega^{s}} \frac{(1-x)^{2}}{2}\left|\frac{\partial u}{\partial x}\right|^{2} d x d t \\
+ & \left.\int_{0}^{1}\left(\frac{(1-x)^{2}}{2}\left|\frac{\partial u}{\partial x}\right|^{2}+|u|^{2}\right) d x\right|_{t=s} \\
\leq & K^{2}\left[\int_{Q} \frac{(1-x)^{2}}{2}|f|^{2} d x d t+\int_{0}^{1}\left(\frac{(1-x)^{2}}{2}\left|\frac{\partial \varphi}{\partial x}\right|^{2}+|\varphi|^{2}\right) d x\right] .
\end{aligned}
$$

If we drop the second term in the last inequality and by taking the least upper bound of the left side with respect to $s$ from 0 to $T$, we get the desired estimate (3.1) with $K^{2}=$ $\sigma+\frac{4+2 \sigma+4 b^{2} \sigma}{a_{0}^{2}}$.

Then the uniqueness of the strong solution results from the desired estimate (3.1) and (2.5) holds.

This last inequality implies the following corollaries.

Corollary 3.1 If a strong solution of (2.1)-(1.4) exists, it is unique and continuously depends on $F=(f, \varphi)$.

Proof First, If $u_{1}$ and $u_{2}$ are two solutions of (2.1)-(1.4), then $u=u_{1}-u_{2}$ is a solution of the problem

$$
\begin{cases}£ \sigma=\frac{\partial u}{\partial t}-\frac{\partial}{\partial x}\left(a \frac{\partial u}{\partial x}\right)=0 & \text { for }(x, t) \in] 0,1[\times] 0, T[, \\ l u=u(x, 0)=0, & \text { for } x \in[0,1], \\ u(0, t)=0, & \text { for } t \in[0, T], \\ \int_{0}^{\alpha} \sigma(x, t) d x+\int_{\beta}^{1} \sigma(x, t) d x=0, & 0 \leq \alpha \leq \beta<1, \forall t \in[0, T],\end{cases}
$$

then from Theorem 3.1, we deduce that $\|u\|_{\mathbb{E}} \leq 0$, which implies that $u_{1}=u_{2}$.

Corollary 3.2 The range $R(\bar{L})$ of $\bar{L}$ is closed in $\mathbb{F}$ and $\overline{R(L)}=R(\bar{L})$.

Proof First, we prove that $R(\bar{L})$ is closed. Let $T \in \overline{R(\bar{L})}$, then there exists a sequence $U_{n} \in D(\bar{L})$ such that $\bar{L} U_{n} \underset{n \rightarrow \infty}{\longrightarrow} T$, in $\mathbb{F}$. since $\|U\|_{\mathbb{E}} \leq c\|\bar{L} U\|_{\mathbb{F}}, \forall U \in D(\bar{L})$. Then $\left\|U_{n}\right\|_{\mathbb{E}} \leq$ $c\left\|\bar{L} U_{n}\right\|_{\mathbb{F}}, \forall U_{n} \in D(\bar{L})$, we deduce that the convergence of $\bar{L} U_{n}$ in $\mathbb{F}$ implies the convergence of $U_{n}$ in $\mathbb{E}$, say $U_{n} \underset{n \rightarrow \infty}{\longrightarrow} U$, in $\mathbb{E}$. Since $\bar{L}$ is closed, $\left(U_{n}\right)$ is a sequence in $D(\bar{L})$ and $U_{n} \underset{n \rightarrow \infty}{\longrightarrow} U$, in $\mathbb{E}$, and $\bar{L} U_{n} \underset{n \rightarrow \infty}{\longrightarrow} T$, in $\mathbb{F}$, we have $U \in D(\bar{L})$ and $\bar{L} U=T$, that is, $T \in R(\bar{L})$ Hence, $R(\bar{L})$ is closed in $\mathbb{F}$.

Now, to prove that $R(\bar{L})=\overline{R(L)}$, we observe that $\bar{L}$ is an extension of $L$; therefore, $\Gamma(L) \subset$ $\Gamma(\bar{L})$, where $\Gamma(L)$ is the graph of $L$, hence $R(L) \subseteq R(\bar{L})$, which implies $\overline{R(L)} \subseteq \overline{R(\bar{L})}=R(\bar{L})$. On the other hand, let $T \in R(\bar{L})$, that is, $\bar{L} U=T$ for some $U \in D(\bar{L})$, which means that $(U, \bar{L} U) \in \Gamma(\bar{L})=\overline{\Gamma(L)}$, therefore, there exists a sequence $\left(U_{n}, L U_{n}\right)_{n \in \mathbb{N}}$ in $\Gamma(L)$ such that $\left(U_{n}, L U_{n}\right) \underset{n \rightarrow \infty}{\longrightarrow}(U, T)$ in $\mathbb{E} \times \mathbb{F}$, which implies that $L U_{n} \underset{n \rightarrow \infty}{\longrightarrow} T$ but $U_{n} \in D(L), \forall n \in \mathbb{N}$, then we have $T \in \overline{R(L)}$ and hence $R(\overline{L)} \subset \overline{R(L)}$ 
Corollary 3.2 shows that, to prove that problem (1.1)-(1.4) has a strong solution for arbitrary $F$, it suffices to prove that the set $R(L)$ is dense in $\mathbb{F}$.

\section{Solvability of the linear problem}

In order to prove the solvability of problem (2.1)-(1.4) it is sufficient to show that $R(L)$ is dense in $\mathbb{F}$. The proof is based on the following lemma.

Lemma 4.1 Suppose that the function $a$ and its derivatives are bounded.

Let $u \in D_{0}(L)=\{u \in D(L), u(x, 0)=0\}$. If, for $u \in D_{0}(L)$ and some functions $w \in L^{2}(Q)$, we have

$$
\int_{Q} \theta(x) f \bar{\omega} d x d t=0
$$

where

$$
\theta(x)= \begin{cases}\frac{x^{2}}{\alpha^{2}}, & x \in(0, \alpha), \\ w, & x \in(\alpha, \beta), \\ \frac{(1-x)^{2}}{(1-\beta)^{2}}, & x \in(\beta, 1),\end{cases}
$$

then $w$ vanishes almost everywhere in $\Omega$.

Proof Equality (4.1), can be written as follows:

$$
\int_{Q} \frac{\partial u}{\partial t} \bar{\rho} d x d t=\int_{Q} A(t) u \bar{\rho} d x d t
$$

where

$$
\rho=\theta(x) w
$$

and

$$
A(t) u=\frac{\partial}{\partial x}\left(a(x, t) \frac{\partial u}{\partial x}\right)
$$

We introduce the smoothing operators $J_{\varepsilon}^{-1}=\left(I-\varepsilon \frac{\partial}{\partial t}\right)^{-1}$ and $\left(J_{\varepsilon}^{-1}\right)^{*}=\left(I+\varepsilon \frac{\partial}{\partial t}\right)^{-1}$ from $L^{2}(0, T)$ into the space $H^{1}(0, T)$ with respect to $t$, then these operators provide the solution of the problems:

$$
\begin{cases}u_{\varepsilon}(t)-\varepsilon \frac{\partial u_{\varepsilon}}{\partial t}=u(t), & u_{\varepsilon}(0)=0, \\ v_{\varepsilon}^{*}(t)+\varepsilon \frac{\partial v_{\varepsilon}^{*}}{\partial t}=v(t), & v_{\varepsilon}^{*}(T)=0 .\end{cases}
$$

We also have the following properties: If $g \in D(L)$, then $J_{\epsilon}^{-1} g \in D(L)$ and we have

$$
\begin{cases}\lim \left\|J_{\epsilon}^{-1} g-g\right\|_{L^{2}(0, T)}=0, & \text { for } \varepsilon \rightarrow 0 \\ \lim \left\|\left(J_{\epsilon}^{-1}\right)^{*} g-g\right\|_{L^{2}(0, T)}=0, & \text { for } \varepsilon \rightarrow 0\end{cases}
$$


Substituting the function $u$ in (4.2) by the smoothing function $u_{\varepsilon}$ and using the relation

$$
A(t) u_{\varepsilon}=J_{\varepsilon}^{-1} A(t) u-\varepsilon J_{\varepsilon}^{-1} B_{\epsilon}(t) u_{\varepsilon}
$$

where

$$
B_{\epsilon}(t) u_{\varepsilon}=\frac{\partial A(t)}{\partial t} u_{\epsilon}=\frac{\partial}{\partial x}\left(\frac{\partial a}{\partial t} \frac{\partial u_{\varepsilon}}{\partial x}\right)
$$

we obtain

$$
-\int_{\Omega} u \overline{\frac{\partial \rho_{\epsilon}^{*}}{\partial t}} d x d t=\int_{\Omega}\left(A(t) u-\epsilon B_{\varepsilon}(t) u_{\varepsilon}\right) \overline{\rho_{\varepsilon}^{*}} d x d t
$$

Since the operator $A(t)$ has a continuous inverse in $L^{2}(0,1)$ defined by

$$
A^{-1}(t) g=\int_{0}^{x} \frac{d \zeta}{a} \int_{0}^{\zeta} g(\eta) d \eta+C_{1}(t) \int_{0}^{x} \frac{d \zeta}{a}
$$

where the functions $C_{1}(t)$ satisfy the following expression:

$$
C_{1}(t)=\frac{\int_{0}^{1} \frac{K(x)}{a} \int_{0}^{x} g(\eta) d \eta d x}{\int_{0}^{1} \frac{K(x)}{a} d x}
$$

the function $K(x)$ is given by

$$
K(x)= \begin{cases}x-\alpha, & (0, \alpha), \\ 0, & (\alpha, \beta), \\ x-1, & (\beta, 1) .\end{cases}
$$

Then we have $\int_{0}^{\alpha} A^{-1}(t) u d x+\int_{\beta}^{1} A^{-1}(t) u d x=0$, hence, the function $J_{\epsilon}^{-1} u=u_{\varepsilon}$ can be represented in the form

$$
u_{\varepsilon}=J_{\epsilon}^{-1} A^{-1}(t) A(t) u,
$$

then

$$
B_{\varepsilon}(t) g=\frac{\partial^{2} a}{\partial t \partial x} J_{\epsilon}^{-1} \frac{C_{1}(t)+\int_{0}^{\zeta} g(\eta) d \eta}{a}+\frac{\partial a}{\partial t} J_{\epsilon}^{-1} \frac{g}{a}-\frac{\partial a}{\partial t} J_{\epsilon}^{-1} \frac{\frac{\partial a}{\partial x}}{a} \frac{C_{1}(t)+\int_{0}^{\zeta} g(\eta) d \eta}{a} .
$$

Consequently, equality (4.3) becomes

$$
-\int_{\Omega} u \overline{\frac{\partial \rho_{\epsilon}^{*}}{\partial t}} d x d t=\int_{\Omega} A(t) u \overline{h_{\epsilon}} d x d t
$$

where

$$
h_{\epsilon}=\rho_{\epsilon}^{*}-\epsilon B_{\epsilon}^{*}(t) \rho_{\epsilon}^{*}
$$


and

$$
\begin{aligned}
B_{\epsilon}^{*}(t) \rho_{\varepsilon}^{*}= & \frac{1}{a}\left(J_{\epsilon}^{-1}\right)^{*} \frac{\partial a}{\partial t} \rho_{\varepsilon}^{*}-\int_{0}^{x}\left(\frac{1}{a}\left(J_{\epsilon}^{-1}\right)^{*} \frac{\partial^{2} a}{\partial t \partial \zeta} \rho_{\varepsilon}^{*}-\frac{1}{a^{2}} \frac{\partial a}{\partial \zeta}\left(J_{\epsilon}^{-1}\right)^{*} \frac{\partial a}{\partial t} \rho_{\varepsilon}^{*}\right) d \zeta \\
& +\frac{\int_{0}^{1} \frac{K}{a} d x+\int_{x}^{1} \frac{K}{a} d \zeta}{\int_{0}^{1} \frac{K}{a} d x} \int_{0}^{1}\left(\frac{1}{a}\left(J_{\epsilon}^{-1}\right)^{*} \frac{\partial^{2} a}{\partial t \partial \zeta} \rho_{\varepsilon}^{*}-\frac{1}{a^{2}} \frac{\partial a}{\partial \zeta}\left(J_{\epsilon}^{-1}\right)^{*} \frac{\partial a}{\partial t} \rho_{\varepsilon}^{*}\right) d x .
\end{aligned}
$$

The left-hand side of (4.4) is a continuous linear functional of $u$, hence the function $h_{\epsilon}$ has the derivatives $\frac{\partial h_{\epsilon}}{\partial x}, \frac{\partial^{2} h_{\epsilon}}{\partial x^{2}} \in L^{2}(Q)$ and the following conditions are satisfied:

$$
h_{\epsilon}(0, t)=h_{\epsilon}(\alpha, t)=h_{\epsilon}(\beta, t)=h_{\epsilon}(1, t)=0, \quad \frac{\partial h_{\epsilon}}{\partial x}(1, t)=0 .
$$

For a sufficiently small $\epsilon$ and the operator $\frac{\left(U_{\epsilon}^{-1}\right)^{*} \frac{\partial a}{\partial t}}{a}$ is bounded in $L^{2}(Q)$, we have $\left\|\epsilon \frac{\left(U_{\epsilon}^{-1}\right)^{*} \frac{\partial a}{\partial t}}{a}\right\|_{L^{2}(\Omega)}<1$, hence, the operator $I-\epsilon \frac{\left(\mathcal{U}_{\epsilon}^{-1}\right)^{*} \frac{\partial a}{\partial t}}{a}$ has a bounded inverse in $L^{2}(Q)$, we deduce that $\frac{\partial \rho_{\epsilon}^{*}}{\partial x}, \frac{\partial^{2} \rho_{\epsilon}^{*}}{\partial x^{2}} \in L^{2}(Q)$ and the following conditions are satisfied:

$$
\left\{\begin{array}{l}
\rho_{\epsilon}^{*}(0, t)=\rho_{\epsilon}^{*}(\alpha, t)=\rho_{\epsilon}^{*}(\beta, t)=\rho_{\epsilon}^{*}(1, t)=0, \\
\frac{\partial \rho_{\epsilon}^{*}}{\partial x}(\alpha, t)=\frac{\partial \rho_{\epsilon}^{*}}{\partial x}(\beta, t)=\frac{\partial \rho_{\epsilon}^{*}}{\partial x}(1, t)=0 .
\end{array}\right.
$$

We introduce the function $v$ such that

$$
v= \begin{cases}\frac{x}{\alpha} w+\frac{1}{\alpha} \int_{\alpha}^{x} w d \zeta, & x \in(0, \alpha), \\ w, & x \in(\alpha, \beta), \\ v=\frac{1-x}{1-\beta} w-\frac{1}{1-\beta} \int_{\beta}^{x} w d \zeta, & x \in(\beta, 1),\end{cases}
$$

then the function $\rho(x)$ can be expressed as follows:

$$
\rho(x)= \begin{cases}\frac{x^{2}}{\alpha^{2}} w=\frac{x}{\alpha} \nu-\frac{1}{\alpha} \int_{\alpha}^{x} v, & x \in(0, \alpha), \\ w=v, & x \in(\alpha, \beta), \\ \frac{(1-x)^{2}}{(1-\beta)^{2}} w=\frac{1-x}{1-\beta} v+\frac{1}{1-\beta} \int_{\beta}^{x} v, & x \in(\beta, 1) .\end{cases}
$$

Then we deduce that

$$
\left\{\begin{array}{l}
v(0, t)=v(\alpha, t)=v(\beta, t)=v(1, t)=0, \quad \int_{0}^{\alpha} v d x+\int_{\beta}^{1} v d x=0, \\
\frac{\partial v}{\partial x}(\alpha, t)=\frac{\partial v}{\partial x}(\beta, t)=\frac{\partial v}{\partial x}(1, t)=0,
\end{array}\right.
$$

and

$$
\frac{\partial \rho}{\partial x}=H(x) \frac{\partial v}{\partial x}, \quad \text { where } H(x)= \begin{cases}\frac{x}{\alpha}, & x \in(0, \alpha), \\ 1, & x \in(\alpha, \beta) \\ \frac{1-x}{1-\beta}, & x \in(\beta, 1) .\end{cases}
$$

Putting

$$
u=\int_{0}^{t} \exp (c \tau) v d t
$$


in (4.2) and integrating with respect to $x$ and $t$, using (4.5) we obtain

$$
\begin{aligned}
& \operatorname{Re} \int_{Q} A(t) u \bar{\rho} d x d t \\
& \left.\quad=-\int_{\Omega} \frac{H(x)}{2}\left(c a-\frac{\partial a}{\partial t}\right) e^{-c t}\right)\left|\frac{\partial u}{\partial x}\right|^{2} d x d t-\left.\int_{0}^{1} \frac{H(x)}{2} a e^{-c t}\left|\frac{\partial u}{\partial x}\right|^{2} d x\right|_{t=T}
\end{aligned}
$$

and

$$
\operatorname{Re} \int_{0}^{s} \int_{0}^{\alpha} \frac{\partial u}{\partial t} \bar{\rho} d x d t=\int_{0}^{s} \int_{0}^{\alpha} e^{c t} H(x)|v|^{2} d x d t
$$

as we choose

$$
c>\frac{a_{3}}{a_{0}}
$$

then we get

$$
\int_{Q} \exp (c t) H(x)|v|^{2} d x d t=0
$$

so $v=0$ a.e., which implies $\omega=0$.

Theorem 4.1 The range $R(L)$ of the operator $L$ is dense in $\mathbb{F}$.

Proof Since $\mathbb{F}$ is a Hilbert space, we have $\overline{R(L)}=\mathbb{F}$ if and only if the relation

$$
\int_{Q}(1-x)^{2} f \bar{g} d x d t+\int_{0}^{1}(1-x)^{2} \frac{d \varphi}{d x} \frac{\overline{d \psi}}{d x} d x+\int_{0}^{1} \varphi \bar{\psi} d x=0
$$

for an arbitrary $u \in D(L)$ and $(g, \psi) \in \mathbb{F}$, implies that $g=0$ and $\psi=0$.

Putting $u \in D_{0}(L)$ in (4.6), we conclude from Lemma 4.1 that $(1-x)^{2} g=\theta(x) \omega=0$, a.e. then $g=0$.

Taking $u \in D(L)$ in (4.6) yields

$$
\int_{0}^{1}(1-x)^{2} \frac{d \varphi}{d x} \frac{\overline{d \psi}}{d x} d x+\int_{0}^{1} \varphi \bar{\psi} d x=0
$$

Since the two terms in the previous equality vanish independently and since the range of the trace operator is everywhere dense in Hilbert space with the norm

$$
\int_{0}^{1}(1-x)^{2}\left|\frac{d \varphi}{d x}\right|^{2} d x+\int_{0}^{1}|\varphi|^{2} d x
$$

hence, $\psi=0$. Thus $\overline{R(A)}=\mathbb{F}$.

\section{Study of the nonlinear problem}

This section is devoted to the proof of the existence, uniqueness of the solution of the problem (1.1)-(1.4). 
If the solution of problem (1.1)-(1.4) exists, it can be expressed in the form $\theta=w+U$, where $U$ is a solution of the homogeneous problem

$$
\begin{aligned}
& £ U=\frac{\partial U}{\partial t}-\frac{\partial}{\partial x}\left(a \frac{\partial U}{\partial x}\right)=0, \\
& U_{0}=U(x, 0)=\varphi(x), \\
& U(0, t)=0, \\
& \int_{0}^{\alpha} U(x, t) d x+\int_{\beta}^{1} U(x, t) d x=0,
\end{aligned}
$$

and $w$ is a solution of the problem

$$
\begin{aligned}
& £ w=\frac{\partial w}{\partial t}-\frac{\partial}{\partial x}\left(a \frac{\partial w}{\partial x}\right)=F\left(x, t, w, \frac{\partial w}{\partial x}\right), \\
& w(x, 0)=0 \\
& w(0, t)=0 \\
& \int_{0}^{\alpha} w(x, t) d x+\int_{\beta}^{1} w(x, t) d x=0
\end{aligned}
$$

where $F\left(x, t, w, \frac{\partial w}{\partial x}\right)=f\left(x, t, w+U, \frac{\partial(w+U)}{\partial x}\right)$ satisfying the condition

$$
\left|F\left(x, t, u_{1}, v_{1}\right)-F\left(x, t, u_{2}, v_{2}\right)\right| \leq d\left(\left|u_{1}-u_{2}\right|+\left|v_{1}-v_{2}\right|\right) \quad \text { for all } x, t \in Q
$$

According to Theorem 3.1 and Lemma 4.1, the problem (5.1)-(5.4) has a unique solution that depends continuously on $U_{0} \in V^{1,0}(0,1)$ where $V^{1,0}(0,1)$ is a Hilbert space with the scalar product

$$
(u, v)_{V^{1,0}(0,1)}=\int_{0}^{1}(1-x)^{2} \frac{\partial u}{\partial x} \frac{\overline{\partial v}}{\partial x} d x+\int_{0}^{1} u \bar{v} d x
$$

and with the associated norm

$$
\|u\|_{V^{1,0}(0,1)}^{2}=\int_{0}^{1}(1-x)^{2}\left|\frac{\partial u}{\partial x}\right|^{2} d x+\int_{0}^{1}|u|^{2} d x
$$

We shall prove that the problem (5.5) - (5.8) has a weak solution by using an approximation process and passing to the limit.

Assume that $v$ and $w \in C^{1}(Q)$, and the following conditions are satisfied:

$$
\left\{\begin{array}{l}
v(x, T)=0, \int_{0}^{\alpha} v(x, t) d x+\int_{\beta}^{1} v(x, t) d x=0, \\
w(x, 0)=0, \quad w(0, t)=0 .
\end{array}\right.
$$

Taking the scalar product in $L^{2}(Q)$ of Eq. (5.5) and the integrodifferential operator

$$
N v=\lambda(1-x) \int_{0}^{x} v d \zeta-x \int_{x}^{1} g(\zeta, t) d \zeta
$$


where

$$
g(\zeta, t)=k \int_{0}^{\zeta} v d \mu-(k-\lambda) \int_{\alpha}^{\zeta} v d \mu+(k-\lambda) \int_{\beta}^{\zeta} v d \mu d \zeta,
$$

by taking the real part, we obtain

$$
\begin{aligned}
H(w, v) & =\operatorname{Re} \int_{Q} F\left(x, t, w, \frac{\partial w}{\partial x}\right) \overline{N v} d x d t \\
& =\operatorname{Re} \int_{Q} \frac{\partial w}{\partial t} \overline{N v} d x d t-\operatorname{Re} \int_{Q} \frac{\partial}{\partial x}\left(a \frac{\partial w}{\partial x}\right) \overline{N v} d x d t .
\end{aligned}
$$

Substituting the expression of $N v$ in the first integral of the right-hand side of (5.11), integrating by parts with respect to $t$, using the condition (5.10), we get

$$
\operatorname{Re} \int_{Q} \frac{\partial w}{\partial t} \overline{N v}=-\operatorname{Re} \int_{Q} w\left(\lambda(1-x) \int_{0}^{x} \frac{\overline{\partial v}}{\partial t} d \zeta+x \int_{x}^{1} \frac{\overline{\partial g}}{\partial t} d \zeta\right) d x d t
$$

Substituting the expression of $N v$ in the second integral of the right hind-side of (5.11), integrating by parts with respect to $x$, using the condition (5.10), we get

$$
\begin{aligned}
& -\operatorname{Re} \int_{Q} \frac{\partial}{\partial x}\left(a \frac{\partial w}{\partial x}\right) \overline{N v} d x d t \\
& =\operatorname{Re} \int_{Q}\left[(2 \lambda-k x) a-\lambda(1-x) \frac{\partial a}{\partial x}\right] w v-\lambda \operatorname{Re} \int_{Q}(1-x) a w \frac{\overline{\partial v}}{\partial x} d x d t \\
& \quad-\operatorname{Re} \int_{Q}\left[2 a+x \frac{\partial a}{\partial x}\right] w g d x d t+\lambda \operatorname{Re} \int_{Q} \frac{\partial a}{\partial x} w \int_{0}^{\zeta} v d \mu d x d t \\
& \quad-\operatorname{Re} \int_{Q} \frac{\partial a}{\partial x} w \int_{x}^{1} \bar{g} d \zeta d x d t .
\end{aligned}
$$

Insertion of (5.12), (5.13) into (5.11) yields

$$
\begin{aligned}
H(w, v)= & -\operatorname{Re} \int_{Q} w\left(\lambda(1-x) \int_{0}^{x} \frac{\overline{\partial v}}{\partial t} d \zeta-x \int_{x}^{1} \frac{\overline{\partial g}}{\partial t} d \zeta\right) d x d t \\
& -\lambda \operatorname{Re} \int_{Q}(1-x) a w \frac{\bar{\partial} v}{\partial x} d x d t+\operatorname{Re} \int_{Q}\left[(2 \lambda-k x) a-\lambda(1-x) \frac{\partial a}{\partial x}\right] w v \\
& -\operatorname{Re} \int_{Q}\left[2 a+x \frac{\partial a}{\partial x}\right] w g d x d t+\lambda \operatorname{Re} \int_{Q} \frac{\partial a}{\partial x} w \int_{0}^{\zeta} v d \mu d x d t \\
& -\operatorname{Re} \int_{Q} \frac{\partial a}{\partial x} w \int_{x}^{1} \bar{g} d \zeta d x d t
\end{aligned}
$$

where

$$
\begin{aligned}
H(w, v)= & \lambda \operatorname{Re} \int_{Q} \bar{v} \int_{x}^{1}(1-\zeta) F\left(\zeta, t, w, \frac{\partial w}{\partial \zeta}\right) d \zeta \\
& -\operatorname{Re} \int_{Q} g \int_{0}^{x} \zeta F\left(\zeta, t, w, \frac{\partial w}{\partial \zeta}\right) d \zeta d x d t
\end{aligned}
$$

obtained by integrating by parts the right-hand side of (5.11) with respect to $x$. 
Definition 5.1 By a weak solution of problem (5.5)-(5.8) we mean a function $w \in L^{2}(0, T$ : $\left.V^{1,0}(0,1)\right)$ satisfying the identity $(5.14)$ and the integral condition (5.8).

We will construct an iteration sequence in the following way.

Starting with $w_{0}=0$, the sequence $\left(w_{n}\right)_{n \in \mathbb{N}}$ is defined as follows: given $w_{n-1}$, then, for $n \geq 1$, we solve the problem

$$
\begin{aligned}
& £ w_{n}=\frac{\partial w_{n}}{\partial t}-\frac{\partial}{\partial x}\left(a \frac{\partial w_{n}}{\partial x}\right)=F\left(x, t, w_{n-1}, \frac{\partial w_{n-1}}{\partial x}\right), \\
& w_{n}(x, 0)=0 \\
& w_{n}(0, t)=0 \\
& \int_{0}^{\alpha} w_{n}(x, t) d x+\int_{\beta}^{1} w_{n}(x, t)=0 .
\end{aligned}
$$

From Theorem 3.1 and Lemma 4.1, we deduce that, for fixed $n$, each problem (5.15)-(5.18) has a unique solution $w_{n}(x, t)$. If we set $V_{n}(x, t)=w_{n+1}(x, t)-w_{n}(x, t)$, we obtain the new problem

$$
\begin{aligned}
& £ V_{n}=\frac{\partial V_{n}}{\partial t}-\frac{\partial}{\partial x}\left(a \frac{\partial V_{n}}{\partial x}\right)=\sigma_{n-1}, \\
& V_{n}(x, 0)=0, \\
& V_{n}(0, t)=0 \\
& \int_{0}^{\alpha} V_{n}(x, t) d x+\int_{\beta}^{1} V_{n}(x, t)=0,
\end{aligned}
$$

where

$$
\sigma_{n-1}=F\left(x, t, w_{n}, \frac{\partial w_{n}}{\partial x}\right)-F\left(x, t, w_{n-1}, \frac{\partial w_{n-1}}{\partial x}\right) .
$$

Theorem 5.1 Assume that the condition (5.9) holds, for the linearized problem (5.19)(5.22), there exists a positive constant $k$, such that

$$
\left\|V_{n}\right\|_{L^{2}\left(0, T: V^{1,0}(0,1)\right)} \leq k\left\|V_{n-1}\right\|_{L^{2}\left(0, T: V^{1,0}(0,1)\right)} .
$$

Proof We denote

$$
M \frac{\partial V_{n}}{\partial t}=\frac{(1-x)^{2}}{2} \frac{\partial V_{n}}{\partial t}+\lambda(1-x) \int_{0}^{x} \frac{\partial V_{n}}{\partial t} d \zeta-x e^{\delta(x-1)} \int_{x}^{1} g(\zeta, t) d \zeta
$$

where

$$
g(\zeta, t)=k \int_{0}^{\zeta} \frac{\partial V_{n}}{\partial t} d \mu-(k-\lambda) \int_{\alpha}^{\zeta} \frac{\partial V_{n}}{\partial t} d \mu+(k-\lambda) \int_{\beta}^{\zeta} \frac{\partial V_{n}}{\partial t} d \mu d \zeta,
$$

and $\lambda, k$ and $\delta$ are scalars parameters such that

$$
3<\frac{\lambda}{k}<4 e^{-\delta} \quad \text { with } \delta>\ln \left(\frac{4}{3}\right) .
$$


We consider the quadratic form obtained by multiplying Eq. (5.19) by $e^{-c t} \overline{M \frac{\partial V_{n}}{\partial t}}$, with the constant $c$ satisfying (3.11), integrating over $Q_{s}=[0,1] \times[0, s]$, with $0 \leq s \leq T$, taking the real part, we obtain

$$
\begin{aligned}
\Phi\left(V_{n}, V_{n}\right)= & \operatorname{Re} \int_{Q_{s}} e^{-c t} \sigma_{n-1} \overline{M \frac{\partial V_{n}}{\partial t}} d x d t \\
= & \operatorname{Re} \int_{Q_{s}} e^{-c t} x^{2}(1-x)^{2} \frac{\partial V_{n}}{\partial t} \overline{M \frac{\partial V_{n}}{\partial t}} d x d t \\
& -\operatorname{Re} \int_{Q_{s}} e^{-c t} \frac{\partial}{\partial x}\left(a \frac{\partial V_{n}}{\partial x}\right) \overline{M \frac{\partial V_{n}}{\partial t}} d x d t .
\end{aligned}
$$

Integrating with respect to $x$ and $t$, using the conditions (5.20), (5.21) and (5.22) we get

$$
\begin{aligned}
\frac{\lambda}{2} \int_{Q^{s}} & \frac{(1-x)^{2}}{2} \exp (-c t)\left|\frac{\partial V_{n}}{\partial t}\right|^{2} d x d t+\frac{\lambda}{2} \int_{Q^{s}}\left(c a-\frac{\partial a}{\partial t}\right) \frac{(1-x)^{2}}{2} e^{-c t}\left|\frac{\partial V_{n}}{\partial x}\right|^{2} d x d t \\
& +\frac{1}{2 k} \int_{Q^{s}}\left(\frac{\lambda}{k}-2 x e^{\delta(x-1)}\right) e^{-c t}|g(x, t)|^{2} d x d t+\frac{\lambda}{2 k^{2}} \int_{Q^{s}} e^{-c t}|g(0, t)|^{2} d x d t \\
& +\frac{1}{2 k} e^{-\delta} \int_{0}^{s} e^{-c t}\left|\int_{0}^{1} g(x, t)\right|^{2} d x d t \\
& +\frac{1}{2} \int_{Q^{s}}\left(c a-\frac{\partial a}{\partial t}\right)\left(\lambda-k x e^{\delta(x-1)}\right) e^{-c t}\left|V_{n}\right|^{2} d x d t \\
& +\frac{1}{2 k} \int_{Q^{s}}\left(2 \delta+\delta^{2} x\right) e^{\delta(x-1)} e^{-c t}\left|\int_{x}^{1} g(\zeta, t) d \zeta\right|^{2} d x d t \\
& +\left.\frac{\lambda}{2} \int_{0}^{1} \frac{(1-x)^{2}}{2} e^{-c s} a(x, s)\left|\frac{\partial V_{n}}{\partial x}\right|^{2}\right|_{t=s} d x \\
& +\left.\frac{1}{2} \int_{0}^{1}\left(\lambda-k x e^{\delta(x-1)}\right) e^{-c s} a(x, s)\left|V_{n}\right|^{2}\right|_{t=s} d x \\
& +\lambda \operatorname{Re} \int_{Q^{s}} \frac{\partial a(x, t)}{\partial x} e^{-c t} V_{n} \int_{0}^{x} \frac{\partial u}{\partial t} d \zeta d x d t \\
& -\operatorname{Re} \int_{Q^{s}}\left(x \frac{\partial a}{\partial x}+2 a(1+\delta x)\right) e^{\delta(x-1)} e^{-c t} V_{n} \overline{g(x, t)} d x d t \\
& -\frac{\lambda}{2 k^{2}} \operatorname{Re} \int_{0}^{s} e^{-c t} g(0, t) \int_{0}^{1} \bar{g}(x, t) d x d t \\
& -\operatorname{Re} \int_{Q^{s}}\left((1+\delta x) \frac{\partial a}{\partial x}+\left(2 \delta+\delta^{2} x\right) a\right) e^{\delta(x-1)} e^{-c t} V_{n} \int_{x}^{1} g(\zeta, t) d \zeta d x d t \\
& \int_{Q^{s}} \frac{\partial V_{n}}{\partial t} d x d t . \\
&
\end{aligned}
$$

Following the same procedure as performed in establishing the proof of Theorem 3.1, we get

$$
\int_{0}^{T} \int_{0}^{1}\left(\frac{(1-x)^{2}}{2}\left|\frac{\partial V_{n}}{\partial x}\right|^{2}+\left|V_{n}\right|^{2}\right) d x \leq K \int_{Q} \frac{(1-x)^{2}}{2}\left|\sigma_{n-1}\right|^{2} d x d t
$$


where

$$
K=\frac{\max \left(\left(\frac{2 k^{2}}{\lambda^{2}-3 k}+\frac{k^{2}}{128 \lambda}+66 \lambda\right)\right)}{\min \left(M, \frac{\lambda-k}{2} e^{-\delta}\left(c a_{0}-c_{2}\right)\right)} e^{c T},
$$

using (5.9), the above inequality becomes

$$
\left\|V_{n}\right\|_{L^{2}\left(0, T: V^{1,0}(0,1)\right)}^{2} \leq k^{2}\left\|V_{n-1}\right\|_{L^{2}\left(0, T: V^{1,0}(0,1)\right)}^{2}
$$

where

$$
k^{2}=2 d^{2} \frac{\frac{2 k^{2}}{\lambda^{2}-3 k}+\frac{k^{2}}{128 \lambda}+66 \lambda}{\min \left(M, \frac{\lambda-k}{2} e^{-\delta}\left(c a_{0}-c_{2}\right)\right)} e^{c T} .
$$

From the criterion of convergence of the series, we see that the series $\sum_{n \geq 1} V_{n}(x, t)$ converges if

$$
d^{2}<\frac{1}{2} \frac{\min \left(M, \frac{\lambda-k}{2} e^{-\delta}\left(c a_{0}-c_{2}\right)\right)}{\frac{2 k^{2}}{\lambda^{2}-3 k}+\frac{k^{2}}{128 \lambda}+66 \lambda} e^{-c T} .
$$

Since $V_{n}(x, t)=w_{n+1}(x, t)-w_{n}(x, t)$, it follows that the sequence $w_{n}(x, t)$ defined by

$$
w_{n}(x, t)=\sum_{k=1}^{k=n-1} V_{k}+w_{0}(x, t)
$$

converges to an element $w \in L^{2}\left(0, T: V^{1,0}(0,1)\right)$. Now to prove that this limit function $w$ is a solution of the problem under consideration (5.19)-(5.22), we should show that $w$ satisfies (5.8) and (5.14).

For problem (5.15)-(5.18), we have

$$
\begin{aligned}
& H\left(w_{n}-w, v\right)+H(w, v) \\
& =\lambda \operatorname{Re} \int_{Q} \bar{v} \int_{x}^{1}(1-\eta)\left(F\left(\eta, t, w_{n-1}, \frac{\partial w_{n-1}}{\partial \eta}\right)-F\left(\eta, t, w, \frac{\partial w}{\partial \eta}\right) d \eta\right) d x d t \\
& \quad-\operatorname{Re} \int_{Q} g \int_{0}^{x} \eta\left(F\left(\eta, t, w_{n-1}, \frac{\partial w_{n-1}}{\partial \eta}\right)-F\left(\eta, t, w, \frac{\partial w}{\partial \eta}\right)\right) d \eta d x d t \\
& \quad+\lambda \operatorname{Re} \int_{Q} \bar{v} \int_{x}^{1}(1-\zeta) F\left(\zeta, t, w, \frac{\partial w}{\partial \zeta}\right) d \zeta \\
& \quad-\operatorname{Re} \int_{Q} g \int_{0}^{x} \zeta F\left(\zeta, t, w, \frac{\partial w}{\partial \zeta}\right) d \zeta d x d t .
\end{aligned}
$$

From Eq. (5.15),we have

$$
\begin{aligned}
H\left(w_{n}-w, v\right)= & \operatorname{Re} \int_{Q} \frac{\partial\left(w_{n}-w\right)}{\partial t}\left(\lambda(1-x) \int_{0}^{x} \bar{v} d \zeta-x \int_{x}^{1} \bar{g}(\zeta, t) d \zeta\right) d x d t \\
& -\operatorname{Re} \int_{Q} \frac{\partial}{\partial x}\left(a \frac{\partial\left(w_{n}-w\right)}{\partial x}\right)\left(\lambda(1-x) \int_{0}^{x} \bar{v} d \zeta-x \int_{x}^{1} \bar{g}(\zeta, t) d \zeta\right) d x d t
\end{aligned}
$$


Djerad et al. Boundary Value Problems

(2021) 2021:70

Page 21 of 24

Integrating by parts each term in the previous equality with respect to $t$ and $x$ using the condition (5.10), we obtain

$$
\begin{aligned}
& H\left(w_{n}-w, v\right) \\
& =-\operatorname{Re} \int_{Q}\left(w_{n}-w\right)\left(\lambda(1-x) \int_{0}^{x} \frac{\partial v}{\partial t} d \zeta-x \int_{x}^{1} \frac{\overline{\partial g}}{\partial t}(\zeta, t) d \zeta\right) d x d t \\
& \quad-\lambda \operatorname{Re} \int_{Q}(1-x) a\left(w_{n}-w\right) \frac{\partial v}{\partial x} d x d t \\
& \quad+\operatorname{Re} \int_{Q}\left[(2 \lambda-k x) a-\lambda(1-x) \frac{\partial a}{\partial x}\right]\left(w_{n}-w\right) v d x d t \\
& \quad-\operatorname{Re} \int_{Q}\left[2 a+x \frac{\partial a}{\partial x}\right]\left(w_{n}-w\right) g d x d t+\lambda \operatorname{Re} \int_{Q} \frac{\partial a}{\partial x}\left(w_{n}-w\right) \int_{0}^{\zeta} v d \mu d x d t \\
& \quad-\operatorname{Re} \int_{Q} \frac{\partial a}{\partial x}\left(w_{n}-w\right) \int_{x}^{1} \bar{g} d \zeta d x d t,
\end{aligned}
$$

where each term of the left-hand side of (5.28) is controlled by

$$
\begin{aligned}
& -\operatorname{Re} \int_{Q}\left(w_{n}-w\right)\left(\lambda(1-x) \int_{0}^{x} \frac{\overline{\partial v}}{\partial t} d \zeta-x \int_{x}^{1} \frac{\overline{\partial g}}{\partial t}(\zeta, t) d \zeta\right) d x d t \\
& \leq 2 \sqrt{2} \max (\lambda, 1)\left(\int_{Q}\left|w_{n}-w\right|^{2} d x d t\right)^{\frac{1}{2}}\left(\int_{Q}(1-x)^{2}\left|\frac{\partial v}{\partial t}\right|^{2} d x d t+\int_{Q}\left|\frac{\partial g}{\partial t}\right|^{2} d x d t\right)^{\frac{1}{2}} \\
& \operatorname{Re} \int_{Q}\left[(2 \lambda-k x) a-\lambda(1-x) \frac{\partial a}{\partial x}\right]\left(w_{n}-w\right) v d x d t \\
& \leq\left((2 \lambda-k) a_{1}+\lambda b\right)\left(\int_{Q}\left|w_{n}-w\right|^{2} d x d t\right)^{\frac{1}{2}}\left(\int_{Q}|v|^{2} d x d t\right)^{\frac{1}{2}} \\
& -\lambda \operatorname{Re} \int_{Q}(1-x) a\left(w_{n}-w\right) \frac{\overline{\partial v}}{\partial x} d x d t \\
& \leq \lambda a_{1}\left(\int_{Q}\left|w_{n}-w\right|^{2} d x d t\right)^{\frac{1}{2}}\left(\int_{Q}(1-x)^{2}\left|\frac{\partial v}{\partial x}\right|^{2} d x d t\right)^{\frac{1}{2}}, \\
& -\operatorname{Re} \int_{Q}\left[2 a+x \frac{\partial a}{\partial x}\right]\left(w_{n}-w\right) g d x d t \\
& \leq\left(2 a_{1}+b\right) \int_{Q}\left|w_{n}-w\right|^{2} d x d t^{\frac{1}{2}}\left(\int_{Q}|g|^{2} d x d t\right)^{\frac{1}{2}} \\
& \lambda \operatorname{Re} \int_{Q} \frac{\partial a}{\partial x}\left(w_{n}-w\right) \int_{0}^{\zeta} v d \mu d x d t \\
& \leq \lambda b \int_{Q}\left|w_{n}-w\right|^{2} d x d t^{\frac{1}{2}}\left(\int_{Q}|v|^{2} d x d t\right)^{\frac{1}{2}}+\operatorname{Re} \int_{Q} \frac{\partial a}{\partial x}\left(w_{n}-w\right) \int_{x}^{1} \bar{g} d \zeta d x d t \\
& \leq b\left(\int_{Q}\left|w_{n}-w\right|^{2} d x d t\right)^{\frac{1}{2}}\left(\int_{Q}\left|\frac{\partial g}{\partial t}\right|^{2} d x d t\right)^{\frac{1}{2}} .
\end{aligned}
$$


From the previous inequalities, we deduce that

$$
\begin{aligned}
\left|H\left(w_{n}-w, v\right)\right| \leq & C\left\|w_{n}-w\right\|_{L^{2}\left(0, T: V^{1,0}(0,1)\right)}\left[\int_{Q}\left((1-x)^{2}\left(\left|\frac{\partial v}{\partial x}\right|^{2}+\left|\frac{\partial v}{\partial t}\right|^{2}\right)+|v|^{2}\right) d x d t\right. \\
& \left.+\int_{Q}\left(\left|\frac{\partial g}{\partial t}\right|^{2}+|g|^{2}\right) d x d t\right]^{\frac{1}{2}}
\end{aligned}
$$

where

$$
C=\max \left\{2 \sqrt{2} \max (\lambda, 1),(2 \lambda-k) a_{1}+\lambda b, \lambda a_{1},\left(2 a_{1}+b\right)\right\} .
$$

Using the condition (5.9) and the Cauchy-Schwarz inequality in the first two terms in the left-hand side in (5.27), we get

$$
\begin{aligned}
& \lambda \operatorname{Re} \int_{Q} \bar{v} \int_{x}^{1}(1-\zeta)\left[F\left(\eta, t, w_{n-1}, \frac{\partial w_{n-1}}{\partial \eta}\right)-F\left(\zeta, t, w, \frac{\partial w}{\partial \zeta}\right)\right] d \zeta \\
& \quad-\operatorname{Re} \int_{Q} g \int_{0}^{x} \zeta\left[F\left(\eta, t, w_{n-1}, \frac{\partial w_{n-1}}{\partial \eta}\right)-F\left(\zeta, t, w, \frac{\partial w}{\partial \zeta}\right)\right] d \zeta d x d t \\
& \leq(\lambda+1) d\left\|w_{n}-w\right\|_{L^{2}\left(0, T: V_{0}^{1}(0,1)\right)}\left(\int_{Q}|v|^{2} d x d t+\int_{Q}|g|^{2} d x d t\right)^{\frac{1}{2}} .
\end{aligned}
$$

From (5.29), (5.30) and passing to the limit in (5.27) as $n \rightarrow+\infty$, we deduce that

$$
\begin{aligned}
H(w, v)= & \lambda \operatorname{Re} \int_{Q} \bar{v} \int_{x}^{1}(1-\zeta) F\left(\zeta, t, w, \frac{\partial w}{\partial \zeta}\right) d \zeta \\
& -\operatorname{Re} \int_{Q} g \int_{0}^{x} \zeta F\left(\zeta, t, w, \frac{\partial w}{\partial \zeta}\right) d \zeta d x d t
\end{aligned}
$$

Now we show that (5.8) holds. Since $\lim _{n \rightarrow+\infty}\left\|w_{n}-w\right\|_{L^{2}\left(0, T: V^{1,0}(0,1)\right)}=0$,

$$
\lim _{n \rightarrow+\infty}\left|\int_{0}^{\alpha}\left(w_{n}-w\right) d x+\int_{\beta}^{1}\left(w_{n}-w\right) d x\right|^{2} \leq \lim _{n \rightarrow+\infty} \int_{0}^{1}\left|w_{n}-w\right|^{2} d x
$$

So

$$
\int_{0}^{\alpha} w d x+\int_{\beta}^{1} w d x=0
$$

Let us now prove the uniqueness of the solution.

Theorem 5.2 If condition (5.9) is satisfied, then the solution of problem (5.5)-(5.8) is unique.

Proof Suppose that $w_{1}, w_{2} \in L^{2}\left(0, T: V^{1,0}(0,1)\right)$ are two solutions of (5.5)-(5.8), the function $v=w_{1}-w_{2}$ is in $L^{2}\left(0, T: V^{1,0}(0,1)\right)$ and satisfies

$$
\frac{\partial v}{\partial t}-\frac{\partial}{\partial x}\left(a \frac{\partial v}{\partial x}\right)=G(x, t)
$$




$$
\begin{aligned}
& v(x, 0)=0, \\
& v(0, t)=0, \\
& \int_{0}^{\alpha} v d x+\int_{\beta}^{1} v d x=0,
\end{aligned}
$$

where $G(x, t)=F\left(x, t, w_{1}, \frac{\partial w_{1}}{\partial x}\right)-F\left(x, t, w_{2}, \frac{\partial w_{2}}{\partial x}\right)$.

Taking the inner product in $L^{2}(Q)$ of Eq. (5.32) and the integro-differential operator

$$
M \frac{\partial v}{\partial t}=\frac{(1-x)^{2}}{2} \frac{\partial v}{\partial t}+\lambda(1-x) \int_{0}^{x} \frac{\partial v}{\partial t} d \zeta-x \int_{x}^{1} g(\zeta, t) d \zeta
$$

where $\lambda$ satisfied (3.2) and following the same procedure as done in establishing the proof of Theorem 3.1, we get

$$
\|v\|_{L^{2}\left(0, T: V^{1,0}(0,1)\right)}^{2} \leq k^{2}\|v\|_{L^{2}\left(0, T: V^{1,0}(0,1)\right)}^{2}
$$

where

$$
k^{2}=\frac{2\left(\frac{2 k^{2}}{\lambda^{2}-3 k}+\frac{k^{2}}{128 \lambda}+66 \lambda\right)}{\min \left(M, \frac{\lambda-k}{2} e^{-\delta}\left(c a_{0}-c_{2}\right)\right)} d^{2} e^{c T} .
$$

Since $k^{2}<1$, we have $v=0$, which implies that $w_{1}=w_{2} \in L^{2}\left(0, T: V^{1,0}(0,1)\right)$.

\section{Acknowledgements}

Not applicable.

Funding

Not applicable.

Availability of data and materials

Not applicable.

\section{Competing interests}

The authors declare that they have no competing interests.

Authors' contributions

All authors contributed equally to the writing of this paper. All authors read and approved the final manuscript.

\section{Authors' information}

Not applicable.

\section{Author details}

'Laboratoire de Mathematiques Pures et Appliquées, Department of Mathematics, University of Msila, Msila, Algeria 28000. ${ }^{2}$ Department of Mathematics, University of Msila, Msila, Algeria 28000. ${ }^{3}$ Department of Mathematics, University of Constantine 1, Constantine, Algeria 25000.

\section{Publisher's Note}

Springer Nature remains neutral with regard to jurisdictional claims in published maps and institutional affiliations.

Received: 8 March 2021 Accepted: 19 July 2021 Published online: 06 August 2021

References

1. Cahlon, B., Kulkarni, D.M., Shi, P.: Stepwise stability for the heat equation with nonlocal constraint. SIAM J. Numer. Anal. 32(2), 571-593 (1995)

2. Cannon, J.R.: The solution of the heat equation subject to the specification of energy. Q. Appl. Math. 21(2), 155-160 (1963)

3. Ionkin, N.I: The solution of a certain boundary value problem of the theory of heat conduction with a nonclasical boundary condition. Differ. Uravn. (Russian) 13(2), 294-304 (1977) 
4. Kamynin, N.I: A boundary value problem in the theory of the heat conduction with non classical boundary conditions. Th. Vychisl. Mat. Fiz 4(6), 1006-1024 (1964)

5. Samarski, A.A.: Some problems in the modern theory of differential equations. Differ. Uravn. (Russian) 16(11), $1221-1228(1980)$

6. Shi, P., Shilor, M.: Design of Contact Patterns in One Dimensional Thermoelasticity in Theoretical Aspects of Industrial Design. SIAM, Philadelphia (1992)

7. Choi, Y.S., Chan, K.Y: A parabolic equation with nonlocal boundary conditions arising from electrochemistry. Nonlinear Anal. 18(4), 317-331 (1992)

8. Cushman, J.H., Xu, H., Deng, F.: Nonlocal reactive transport with physical and chemical heterogeneity: localization error. Water Resour. Res. 31(9), 2219-2237 (1995)

9. Ewing, R.E., Lin, T.: A class of parameter estimation techniques for fluid flow in porous media. Adv. Water Resour. 14(2), 89-97 (1991)

10. Nakhushev, A.M.: On a certain approximate method for boundary-value problems for differential equations and their applications in ground waters dynamics. Differ. Uravn. 18(1), 72-81 (1982)

11. Vogakhova, V.A.: A boundary-value problem with Nakhuchev nonlocal condition for a certain pseudoparabolic water transfer equation. Differ. Uravn. 18(2), 280-285 (1982)

12. Batten, G.W. Jr.: Second-order correct boundary conditions for the numerical solution of mixed boundary problem for parabolic equations. Math. Comput. 17(84), 405-413 (1963)

13. Beilin, S.B.: Existence of solutions for one-'wave equation with a nonlocal condition. Electron. J. Differ. Equ. 2001, Article ID 76 (2001)

14. Cannon, J.R.: The One-Dimensional Heat Equation. Encyclopedia of Mathematics Ant Its Applications, vol. 23. Addison-Wesley, Massachusets (1984)

15. Belmouloud, I., Memou, A.: On the solvability of a class of nonlinear singular parabolic equation with integral boundary condition. Appl. Math. Comput. 373, Article ID 124999 (2020)

16. Kartynnik, A.V:: A three-point boundary-value problem with an integral space-variable condition for a second-order parabolic equations. Differ. Equ. 26(9), 1160-1166 (1990)

17. Shi, P.: Weak solution to evolution problem with a nonlocal constraint. SIAM J. Math. Anal. 24(1), 46-58 (1993)

18. Yurchuk, N.I: Mixed problem with an integral condition for certain parabolic equations. Differ. Equ. 22(12), 1457-1463 (1986)

19. Pulkina, L.S.: A non-local problem with integral conditions for hyperbolic equations. Electron. J. Differ. Equ. 1999, Article ID 45 (1999)

20. Pulkina, L.S.: A non-local problem with integral conditions for the quasilinear hyperbolic equation. Mathematical Notes 70(1), 79-85 (2001)

21. Pulkina, L.S.: A mixed problem with integral condition for the hyperbolic equation. Mathematical Notes 74(3), 411-421 (2003)

22. Volkodavov, V.F., Zhukov, V.E.: Two problems for the string vibration equation with integral conditions and special matching conditions on the characteristic. Differ. Equ. 34(4), 503-507 (1998)

23. Denche, M., Marhoune, A.L.: A three-point boundary value problem with an integral condition for parabolic equations with the Bessel operator. Appl. Math. Lett. 13(6), 85-89 (2000)

24. Denche, M., Marhoune, A.L.: High order mixed-type differential equations with weighted integral boundary conditions. Electron. J. Differ. Equ. 2000, Article ID 60 (2000)

25. Denche, M., Marhoune, A.L.: Mixed problem with nonlocal boundary conditions for a third order partial differential equation of mixed type. Int. J. Math. Math. Sci. 26(7), 417-426 (2001)

26. Denche, M., Marhoune, A.L.: Mixed problem with integral boundary condition for a high order mixed type partia differential equation. J. Appl. Math. Stoch. Anal. 16(1), 69-79 (2003)

27. Latrous, C., Memou, A.: A three-point boundary value problem with an integral condition for a third order partial differential equation. Abstr. Appl. Anal. 2005(1), 33-43 (2005)

28. Gushin, A.K., Mikhailov, V.P.: On solvability of nonlocal problem for second-order elliptic equation. Mat. Sb. 185(1), 121-160 (1994)

29. Skubachevski, A.L., Steblov, G.M.: On spectrum of differential operators with domain non-dense in $/ 2$. Doklad. Akad. Nauk USSR 321(6), 1158-1163 (1991)

30. Paneiah, B.P.: On certain nonlocal boundary problem's for linear differential operators. Mat. Zametki 35(3), 425-434 (1984)

31. Marhoune, A.L., Lakhal, F.: A boundary value problem with multivariables integral type condition for parabolic equations. J. Appl. Math. Stoch. Anal. 2009, Article ID 975601 (2009)

32. Marhoune, A.L., Memou, A.: Nonlocal singular problem with integral condition for a second-order parabolic equation. Electron. J. Differ. Equ. 2015, 64 (2015) 